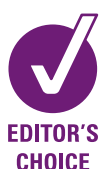

CHOICE

\title{
Cranial dural arteriovenous fistula: transarterial Onyx embolization experience and technical nuances
}

\author{
Yin C Hu, C Benjamin Newman, Shervin R Dashti, Felipe C Albuquerque, \\ Cameron G McDougall
}

Division of Neurological Surgery, Barrow Neurological Institute, St. Joseph's Hospital and Medical Center, Phoenix, Arizona, USA

\section{Correspondence to}

Dr C G McDougall, Division of Neurological Surgery, Barrow Neurological Institute, St. Joseph's Hospital and Medical Center, 2910 N Third Ave, Phoenix, AZ 85013, USA; cameron.mcdougall@bnaneuro. net

Received 20 August 2010 Accepted 7 September 2010

Published Online First

6 January 2011

\section{ABSTRACT}

Introduction Traditional endovascular treatment of cranial dural arteriovenous fistulas (DAVFs) consists of a transarterial approach with n-butylcyanoacrylate (nBCA) or a transvenous approach with coil embolization. The advent of Onyx in the endovascular arsenal potentially offers a high probability of obliteration of these vascular lesions through a purely transarterial route.

Methods A retrospective review of the Barrow Neurological Institute endovascular database between October 2005 and November 2009 highlighted 50 patients with 63 cranial DAVFs that were treated with transarterial Onyx, with and without adjuvant embolysates, for a total of 76 embolization procedures. Hospital records, cerebral angiography and other diagnostic imaging and clinical visits were reviewed. Results At a median follow-up of 5 months (range 0-25 months), complete angiographic cure was obtained in 41 patients with 50 (79\%) DAVFs after transarterial embolization using Onyx combined with other embolysates. When Onyx was used as the sole embolic agent, 32 of 37 DAVFs (87\%) in 29 (of 33) patients achieved angiographic cure. Subgroup analysis showed that by using the middle meningeal artery (MMA) as a conduit for primary Onyx embolization, angiographic cures were achieved in 27 of the 37 DAVFs (73\%). Periprocedural complications occurred in six $(7.9 \%)$ sessions in one patients $(8 \%)$. Only one patient had a permanent complication (2\%) with unimproved cranial nerve palsies

Conclusion Transarterial Onyx embolization of cranial DAVFs, particularly using the MMA as a conduit, is a safe and effective curative therapy.

Intracranial dural arteriovenous fistulas (DAVFs), lesions of the CNS, account for $10-15 \%$ of all intracranial arteriovenous shunting. ${ }^{1}$ DAVFs typically involve meningeal arteries directly connected to the venous system within the dura mater. Until the first description of intracerebral hemorrhage (ICH) related to DAVFs in the early 1970s, all DAVFs were considered benign compared with arteriovenous malformations (AVMs). ${ }^{2-6}$

Decades of anatomical and clinical knowledge of DAVFs have revealed the significance of the location of these lesions, cortical venous reflux (CVR), and their association with adverse clinical presentations. Djindjian and Merland, who proposed the first general classification of DAVFs, emphasized the patterns of venous drainage and their related complications. ${ }^{7}$ In the 1980 s, three large comprehensive reviews dominated the fields of radiographic and clinical characterization of DAVFs. In 1984,
Malik et al reviewed 223 patients and concluded that DAVFs with restricted venous outflow were at a higher risk for hemorrhage and other neurological symptoms compared with lesions draining into large dural sinuses. ${ }^{8}$ In 1986, Lasjaunias et al conducted a meta-analysis of 195 patients and reported that focal CNS symptoms related to CVR were associated with an increased risk of $\mathrm{ICH}^{9}$ Awad et al introduced the 'aggressive' nomenclature to describe DAVFs that manifest with ICH or focal neurologic deficits. ${ }^{10}$ They correlated other high risk angiographic patterns with aggressive neurologic manifestations. These included variceal or aneurysmal venous dilations and leptomeningeal and Galenic venous drainage. A significant conclusion from their study was that no DAVFs were immune from developing aggressive neurologic behavior.

\section{PATHOGENESIS}

The exact pathomechanism for developing DAVFs remains unclear. Most authorities speculate that DAVFs are acquired rather than congenital lesions. There are two main hypotheses about how DAVFs develop. The first theory postulates that these channels are naturally present and become active in the settings of venous hypertension from sinus thrombosis or flow obstruction. ${ }^{11}$ In normal individuals, the presence of these channels has been validated by radiographic and histological findings. ${ }^{12}$ The second theory hypothesizes that these connections are a result of neovascularization in the dura related to released angiogenic factors stimulated by hypoxia or increased venous pressure from venous thrombosis or occlusion. ${ }^{13}$ Findings from histological and animal studies support this observation. ${ }^{13-15}$

\section{CLASSIFICATION SCHEMES}

Numerous classifications had been devised in an attempt to capture the radiological and clinical significance of DAVFs. The initial classification was based on location but subsequent investigations have shown that the clinical behaviors of cavernous and transverse sinus DVAFs differ from those of anterior fossa and tentorial lesions. Borden et al and Cognard et al have proposed the two most practical classifications (table 1). ${ }^{16}{ }^{17}$ Borden type I and Cognard type I and IIa DAVFs are considered benign lesions while the remaining categories are classified as aggressive.

\section{CLINICAL PRESENTATION}

DAVFs can display a spectrum of clinical symptoms depending on their locations, and they can be 
Table 1 Classification nomenclature for dural arteriovenous fistulas

\begin{tabular}{ll}
\hline Classification & Description \\
\hline Borden & \\
Type I & Venous drainage directly into dural sinus or meningeal veins \\
Type II & Venous drainage into dural sinus with CVR \\
Type III & Venous drainage directly into cortical veins only \\
Cognard & \\
Type I & Normal antegrade venous drainage into dural sinus \\
Type Ila & Retrograde venous drainage into dural sinus \\
Type IIb & Retrograde venous drainage into cortical veins (CVR) \\
Type Ila + b & Retrograde venous drainage into dural sinus with CVR \\
Type III & Direct venous drainage into cortical veins without venous ectasia \\
Type IV & Direct venous drainage into cortical veins with venous ectasia \\
& $>5$ mm \\
Type V & Direct venous drainage into spinal perimedullary plexus \\
\hline
\end{tabular}

CVR, cortical venous reflux.

associated with significant morbidity and mortality. Their clinical and angiographic presentations dictate the urgency of treatment (table 2).

Benign DAVFs tend to be found in the cavernous sinus and transverse-sigmoid sinus. Patients with cavernous sinus fistulas
Table 2 Clinical features of cranial dural arteriovenous fistulas

\begin{tabular}{ll}
\hline Clinical group & Manifestation \\
\hline Benign & Pulsatile tinnitus \\
Aggressive & Orbital symptoms \\
& Intracranial hemorrhage \\
& Seizures \\
& Altered mental status \\
& Hydrocephalus \\
& Dementia \\
& Cranial nerve palsies \\
& Loss of motor functions \\
\hline
\end{tabular}

often develop orbital symptoms, including chemosis, proptosis and conjunctival injection. Elevated intraocular pressure related to vascular congestion can lead to decreased visual acuity. Progressive ophthalmoplegia and cranial nerve dysfunction can lead to significant morbidities. Other patients have described pulsatile tinnitus that has devastated their quality of life. The tinnitus is associated with DAVFs with venous drainage into the transverse-sigmoid sinus. This phenomenon most likely reflects
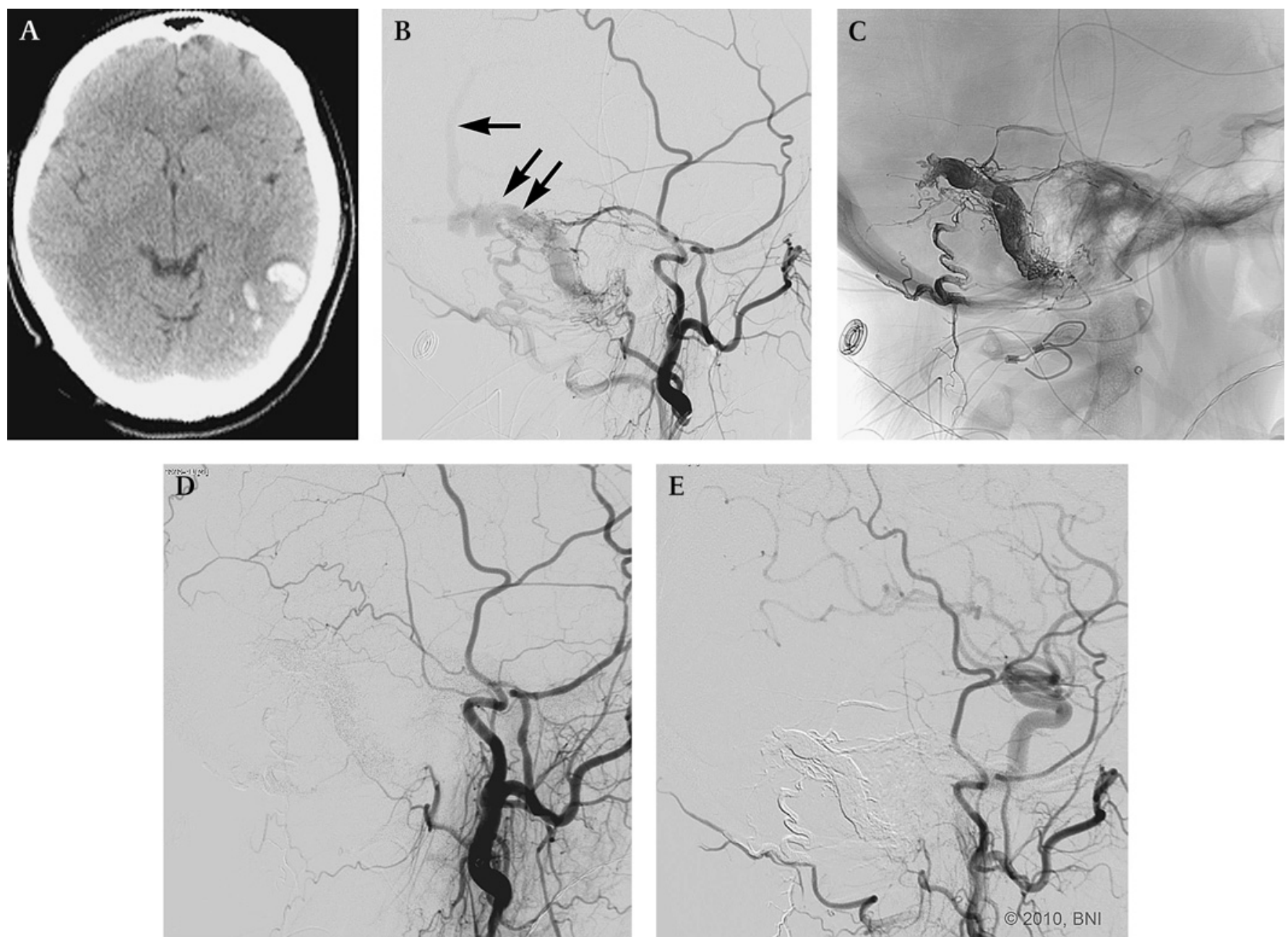

Figure 1 An unresponsive 42-year-old patient with seizure. (A) CT of the head revealed a left posterior temporal intraparenchymal hemorrhage. (B) Lateral view of left external carotid artery injection showed an aggressive dural arteriovenous fistula (DAVF) with an isolated pathologic transverse-sigmoid sinus (double arrows) and cortical venous reflux (single arrow). Arterial feeders can be seen from branches of the middle meningeal artery (MMA), occipital artery and posterior auricular artery. (C) Unsubtracted lateral view showing the Onyx casting from a single infusion via the left MMA resulting in a complete angiographic cure (D). At 6 months (E), no residual DAVF is seen, demonstrating the durability of the Onyx cast. (Used with permission from Barrow Neurological Institute.) 
the proximity of the lesion to the auditory apparatus. Even in these locations, CVR can be demonstrated angiographically and warrants treatment.

Symptomatic aggressive DAVFs may present with hemorrhage, non-hemorrhagic neurological deficits and even death (figure 1). DAVFs with CVR can also demonstrate benign symptoms with subsequent aggressive angiographic features discovered incidentally on cerebral angiography (figure 2). ${ }^{10} 17$ Anterior fossa lesions are fed by ethmoidal arteries and frequently drain into the cavernous sinus. Patients can present with orbital symptoms related to cavernous sinus congestion. However, these lesions have a high predilection for hemorrhage. DAVFs located near the tentorium also have a high propensity for hemorrhages. Superior sagittal sinus DAVFs can exhibit symptoms of global venous congestion such as seizures, hydrocephalus, papilledema and dementia. Patients with brainstem DAVFs often have venous drainage into spinal perimedullary veins and can develop symptoms of lower cranial nerve palsies and quadriparesis from venous congestion and brainstem ischemia.

\section{NATURAL HISTORY}

The ability to treat DAVFs required gaining a thorough understanding of the natural history of the disease and the benefit to risk ratio associated with different treatment modalities. The consensus from studies on the natural history of benign DAVFs was that the behavior of benign DAVFs is stable over time with about $2 \%$ of the lesions converting to aggressive lesions. ${ }^{18}$ The subgroups described as Cognard type II fistulas highlighted the importance of retrograde reflux into the sinus without CVR. Although type IIa lesions are considered benign, progressive thrombosis of the sinus may lead to venous congestion of the cortical veins causing global deficits such as dementia, seizures, parkinsonism and cerebellar symptoms. Despite the stable clinical behaviors ascribed to benign DAVFs, these patients need to be monitored continuously for any changes in symptoms.

The natural history of DAVFs with aggressive features is the subject of debate. Rates of morbidity and mortality have varied due to small samples. Annual rates of subsequent ICH and non-hemorrhagic neurological deficits attributable to aggressive DAVFs have ranged from $1.8 \%$ to $20 \%{ }^{19} 20$ Van Dijk et al followed 20 patients with partially treated or untreated type II or III DAVFs over a mean of 4.3 years. The annual risk for ICH and non-hemorrhagic neurological deficits was $8.1 \%$ and $6.9 \%$, respectively. ${ }^{21}$ Recent publications have suggested that the natural history of DAVFs associated with CVR could be subcategorized based on presenting symptoms. Zipfel et al recently proposed modifiers to the Borden and Cognard classification system to include asymptomatic and symptomatic presentations associated with aggressive DAVFs. ${ }^{22}$ Their proposal was based on studies that patients with symptomatic CVR had an approximately $7.5 \%$ annual risk of ICH (ie, hemorrhages and non-hemorrhagic neurological deficits) compared with a $1.5 \%$ annual risk for those with asymptomatic CVR (ie, ophthalmological symptoms, tinnitus), respectively. ${ }^{23} 24$ These observations are most likely the consequence of flow related and pressure related venous drainage.

\section{THERAPEUTIC CONSIDERATIONS}

Treatment options for DAVFs include endovascular embolization, microsurgery, stereotactic radiosurgery or a combination of
Figure 2 A 62-year-old patient with headaches and left bruit. (A) Lateral view of left external carotid artery (ECA) injection demonstrated an angiographic aggressive dural arteriovenous fistula (DAVF) with arterial feeders from the middle meningeal artery (MMA) and cortical venous reflux (single arrows). (B) Unsubtracted lateral view showing the Onyx casting from a single infusion via the MMA. No residual DAVF is seen on immediate (C) and 6 month follow-up (D) (image is reversed) lateral views of left ECA injections, respectively. (Used with permission from Barrow Neurological Institute.)
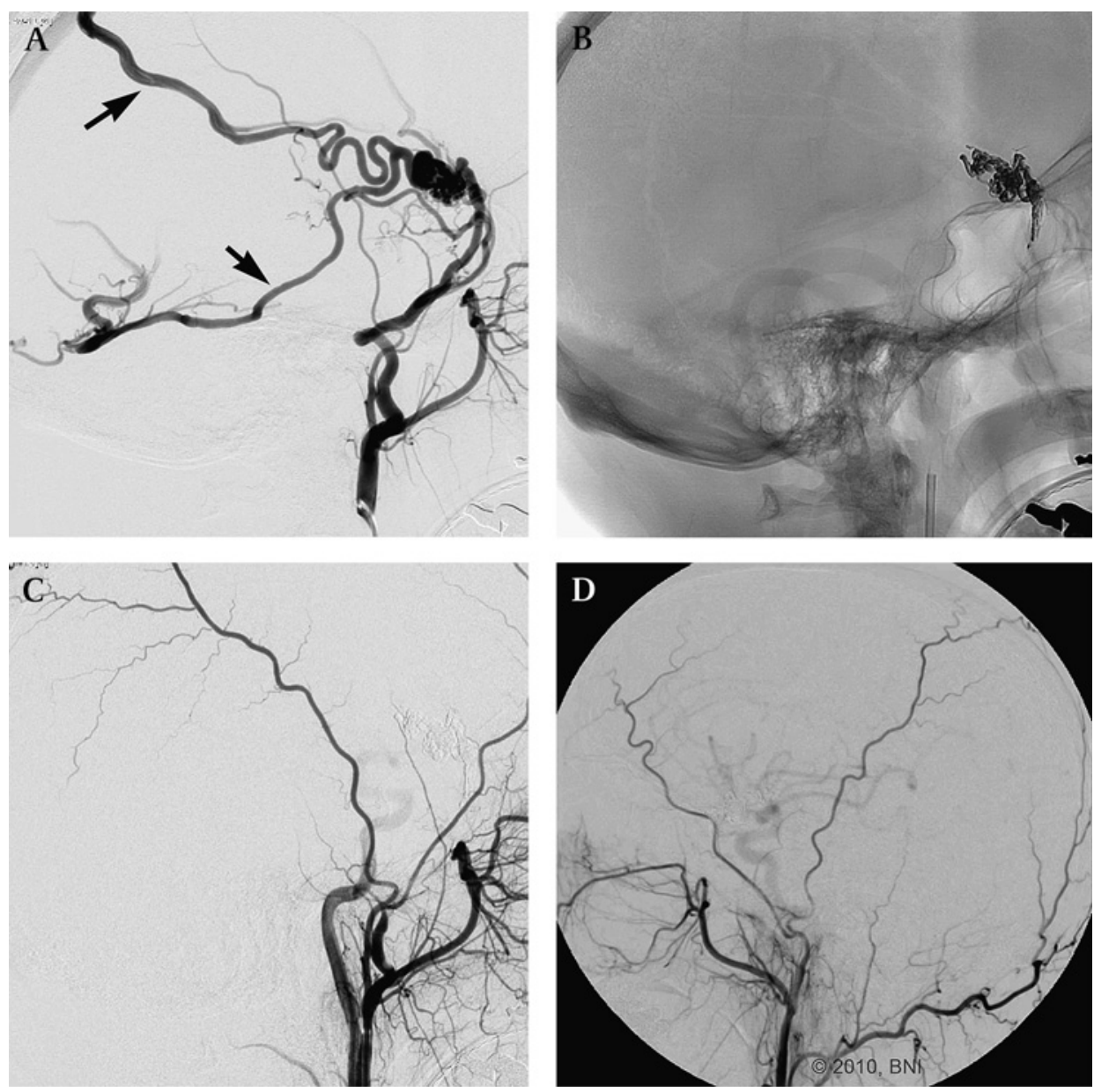
these modalities. Historically, surgical resection of the diseased dura and sinus was the treatment of choice. However, endovascular intervention has quickly evolved and has become the first treatment option for most DAVFs. If endovascular therapies are unsuccessful, microsurgery and radiosurgery can be considered.

\section{Management of DAVFs}

The natural history of benign DAVFs suggests that $98 \%$ of patients have an excellent outcome with only $2 \%$ developing subsequent aggressive conversion. ${ }^{18}$ Clinically stable patients can be managed with observation via serial non-invasive contrast enhanced imaging. Any sudden changes in their clinical symptoms should prompt urgent re-evaluation of their DAVFs to exclude the development of CVR or progressive thrombus with retrograde reflux into the sinus. For patients with lifestyle disabling bruits or severe orbital symptoms, palliative endovascular embolization can be considered. Transarterial or transvenous embolization can significantly reduce or eliminate their symptoms and improve their quality of life.

Aggressive treatment is warranted for DAVFs with CVR because these lesions have a poor natural history. Re-hemorrhage risks have been reported as high as $35 \%$ within 2 weeks of initial hemorrhage. ${ }^{25}$ In most institutions, the first option for therapeutic treatment of DAVFs is endovascular intervention. The main objectives of endovascular therapy are to disconnect the CVR and to occlude the DAVF either through a transarterial, transvenous or a combination of approaches. Various solid (coils and stents) or liquid embolysates (n-butylcyanoacrylate (nBCA),
Onyx), or a combination of these two, can be used. If complete obliteration cannot be achieved or if an endovascular procedure for certain lesions (ie, anterior fossa DAVFs often with ethmoidal feeders from the ophthalmic artery) are associated with considerable risk, microsurgery is an effective alternative method of treatment. Another therapeutic treatment is radiosurgery. Although radiosurgery has been described as a successful modality in small case reports, $1-3$ years must elapse to achieve the desired result. ${ }^{26-28}$ In the interim, there is a possibility that the patient will develop new neurological symptoms. Radiosurgery should not be considered as a primary modality of treatment for cranial DAVFs.

\section{ENDOVASCULAR TECHNIQUES \\ Transvenous approach}

Traditionally, DAVFs have been treated through transvenous coil embolization and/or injections of particles or liquid embolysates (figure 3). Curative rates have been high. Using this approach requires a thorough understanding of the venous drainage. Based on angioarchitectural analysis of transverse and sigmoid sinus DAVFs in cadaveric studies, the DAVFs are drained by parallel venous channels that are clearly separate from the normal cortical venous drainage. ${ }^{29} 30$ If the diseased sinus is isolated from the normal venous circulation, sinus occlusion effectively eliminates the fistula and CVR. The risk for venous infarction is low because normal cortical venous drainage does not depend on the pathological sinus. If a transvenous approach is to be attempted, these distinct channels must be identified to minimize complications.
Figure 3 A 55-year-old patient with a history of intraparenchymal hemorrhage on CT of the head (A) related to a right transverse-sigmoid dural arteriovenous fistula (DAVF). Anteroposterior view (B) of right external carotid artery injection demonstrated an aggressive DAVF, with a parallel venous channel, a venous pouch $\left({ }^{*}\right)$ and cortical venous reflux (CVR) (arrowhead). A transvenous Onyx infusion (C) is performed under a blank roadmap condition. The Onyx cast (double arrows) correlated to the parallel venous channel (single arrow) in (B). Final lateral angiographic view (D) after transarterial and transvenous Onyx infusion demonstrated a very small feeder from the posterior auricular feeder that continued to supply the CVR (arrow). Microsurgical disconnection was performed. (Used with permission from Barrow Neurological Institute.)
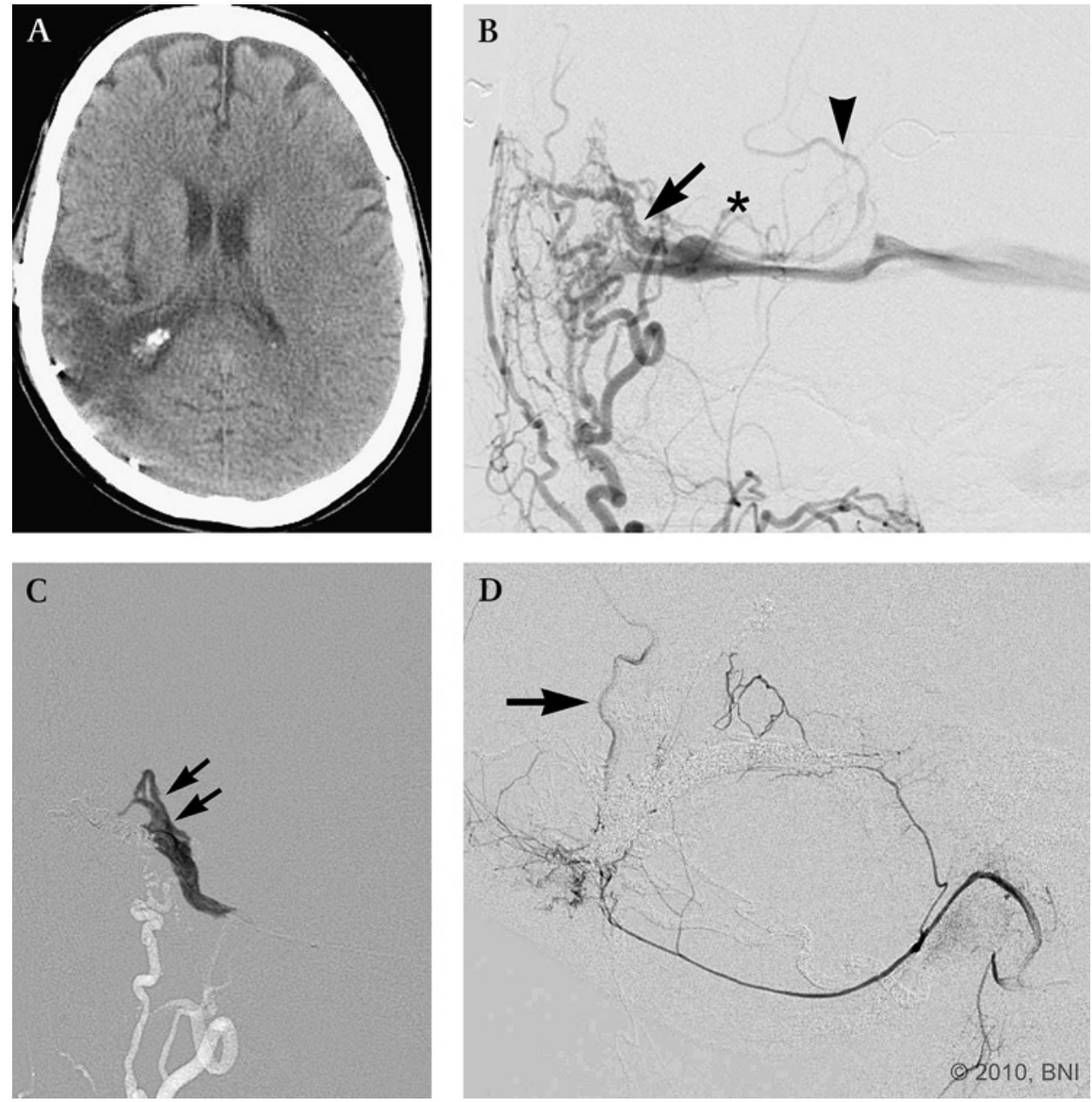


\section{Transarterial approach}

In certain conditions such as venous stenoses or venous occlusion, venous catheterization can be difficult to achieve, rendering this approach almost impossible. Transarterial embolization represented a paradigm shift in the endovascular treatment of DAVFs. This approach requires superselective catheterization of an arterial feeder to deliver the embolysates. In many institutions, the embolysates are liquid agents such as nBCA and Onyx. Complex fistulas might require a multistaged approach with a combination of transvenous and transarterial tactics to disconnect the CVR and occlude the fistula.

In 2005 the Food and Drug Administration approved the use of the novel compound Onyx (eV3, Irvine, California, USA) for the treatment of cerebral AVMs. Onyx is an ethylene vinyl alcohol copolymer preparation suspended in the organic solvent dimethyl sulfoxide (DMSO). Since Onyx was introduced, its therapeutic uses in the treatment of intracranial AVMs have increased due to its deep penetration of the nidus and anecdotal reports of its superior surgical handling properties. However, its off label usages in treating DAVFs are unclear. Recent preliminary reports show high curative rates associated with transarterial embolization with Onyx. Nogueira et al obtained complete angiographic cures in 11 of 12 patients: eight patients after a single procedure and five patients via a single arterial pedicle. ${ }^{31}$ Recently, Cognard et al achieved complete occlusion in 24 of 30 cases of DAVFs associated with CVR by injecting Onyx via transarterial conduits. ${ }^{32}$ These preliminary studies demonstrated positive and promising methodology for treating DAVFs.

At our institution, the approach to treating DAVFs has gradually evolved since Onyx was incorporated into our endovascular armamentarium. By reporting our endovascular experiences and strategies of using transarterial Onyx embolization for treating DAVFs, we hope to delineate potential pitfalls and obstacles that can limit successful treatment and identify tactics that may increase the rate of cure.

\section{BARROW NEUROLOGICAL INSTITUTE EXPERIENCE}

A prospectively collected database of patients referred to the endovascular neurosurgery service at the Barrow Neurological Institute was reviewed. All DAVFs undergoing definitive endovascular treatments with Onyx were included in the analysis; patients undergoing surgical resection of the DAVF were excluded. Patients who failed endovascular treatments and required other therapeutic options were also included. Hospital records, operative reports, cerebral angiograms and radiology reports were reviewed and analyzed. Patients with a DAVF and an additional abnormality such as a tumor, aneurysm or AVM were excluded.

Between October 2005 and November 2009, 50 consecutive patients (35 men and 15 women) underwent 76 embolization procedures involving 63 DAVFs. Of the 76 procedures, Onyx was used as the liquid embolic agent in 70 procedures. The patients' mean age was $56 \pm 13$ years (range $1-82$ years). Clinical presentations included bruit in 22 patients (44\%), intracranial hemorrhage in 13 patients (26\%), headaches in 11 patients $(22 \%)$, mental status change in three patients $(6 \%)$, seizures in three patients $(5 \%)$, weakness in two patients (4\%), cranial nerve palsies in one patient (5\%), visual symptoms in one patient $(2 \%)$, dementia in one patient $(5 \%)$ and dizziness in one patient (5\%). CVR was identified in 36 DAVFs in 29 patients, of which 21 patients presented with aggressive neurologic symptoms, including intracranial hemorrhages, non-hemorrhagic neurological deficits and seizures (table 3 ).
Table 3 Summary of demographics and clinical presentations

\begin{tabular}{ll}
\hline No of patients & 50 \\
Men & 35 \\
Women & 15 \\
Age (years) & \\
Median & $56 \pm 13$ \\
Range & $1-82$ \\
No of DAVFs & 63 \\
Total embolization procedures & 76 \\
Clinical presentations (n (\%)) & \\
Bruit & $22(44)$ \\
Intracranial hemorrhage & $14(22)$ \\
Headache & $11(17)$ \\
Altered mental status & $3(6)$ \\
Seizure & $3(5)$ \\
Progressive weakness & $2(4)$ \\
Cranial nerve palsies & $1(2)$ \\
Visual symptoms & $1(2)$ \\
Dementia & $1(2)$ \\
Dizziness & $1(2)$ \\
Cortical venous reflux (n (\%)) & $36(57)$ \\
Intracranial hemorrhage & $14(39)$ \\
Bruit & $11(31)$ \\
NINDs & $5(14)$ \\
Headaches & $5(14)$ \\
Altered mental status & $2(6)$ \\
Seizure & $2(6)$ \\
\hline DAVF, dural arteriovenous fistula; NIND, non-hemorrhagic neurological \\
deficits.
\end{tabular}

Of the 76 embolization procedures, venous drainage of the DAVF was observed in the following portions of the venous system: transverse-sigmoid sinus $(n=39)$, torcular $(n=22)$, transverse sinus $(n=6)$, superior sagittal sinus $(n=6)$, sigmoid sinus $(n=4)$, occipital sinus $(n=2)$, tentorium $(n=2)$, ethmoidal veins $(n=2)$, pteryoid plexus $(n=1)$, cavernous sinus $(n=1)$ and spinal medullary vein $(n=1)$. The vascular approach for endovascular treatments can be divided into transarterial $(n=54)$, transvenous $(n=3)$ and a combined transarterial and transvenous approach $(\mathrm{n}=19)$.

Onyx was used as the primary embolic agent in 47 of the 76 embolization procedures. In 33 patients with 37 DAVFs, Onyx was used in 39 procedures as the sole embolic agent with no other adjuvant therapies to achieve the final clinical or angiographic results. The middle meningeal artery (MMA) was used in 32 of these 39 procedures. The remaining embolizations required Onyx with assistance from the following adjuvant elements: coils $(n=7) ; n B C A(n=5) ; n B C A$ and coils $(n=2)$; balloons $(n=2)$; balloons and coils $(n=1)$; stents $(n=1)$; and $\mathrm{nBCA}$, coils and stents $(\mathrm{n}=1)$. Transvenous Onyx infusions were performed in four procedures (figure 3). Other embolization procedures (six of 76) did not involve Onyx but were included in this analysis because they were integral parts of staged embolization. These stages included using $\mathrm{nBCA}(\mathrm{n}=1)$, transvenous coils $(n=3)$ and nBCA with coils $(n=2)$ (table 4$)$.

\section{Embolization procedure}

All patients are placed under general anesthesia with neurophysiological monitoring (both somatosensory evoked potentials and electroencephalography) during the embolization treatments in a biplanar fluoroscopic unit. A 6 French sheath is placed percutaneously into the common femoral artery, regardless of the agent used. A baseline activated coagulation time is obtained, and heparin is administered to achieve a targeted 
Table 4 Number and type of embolysates used with Onyx in 76 embolization procedures

\begin{tabular}{ll}
\hline Procedures & Number \\
\hline Transarterial Onyx & 47 \\
Transarterial Onyx + adjuvant therapy & \\
Coiling (total) & 7 \\
Transarterial & 1 \\
Transvenous & 6 \\
Balloon assisted & 3 \\
Balloon assisted with coils & 1 \\
nBCA & 5 \\
nBCA + coiling & 2 \\
nBCA + stenting & 1 \\
nBCA + stenting + coiling & 1 \\
Transvenous Onyx & 1 \\
Transvenous & \\
Onyx & 3 \\
\hline nBCA, n-butylcyanoacrylate. &
\end{tabular}

activated coagulation time of $250-300 \mathrm{~s}$ throughout the procedure. We believe that the risk of rebleeding with periprocedural anticoagulation therapy is low compared with the risks of embolic complications. Standard coaxial techniques were used to catheterize the targeted vessels. A 6 French guide catheter, either an Envoy (Codman, Miami Lakes, Florida, USA) or a Neuron (Penumbra, Alameda, California, USA), depending on the tortuosity of the vessel, is placed in the distal primary targeted vessel.

Arterial pedicles are identified on initial angiographic images. Analysis of the angioarchitecture of the cerebral angiogram were reviewed to evaluate the following critical factors: (1) arterial feeders, (2) fistulous connection, (3) venous outflow, (4) normal dural sinuses, (5) venous anomalies (ie, venous pouches), (6) potential extracranial to intracranial anastomoses and (7) potential anastomoses to cranial nerve vasculatures. The importance of understanding these elements cannot be overemphasized to maximize the therapeutic efficacy of endovascular treatments of DAVFs and to minimize complications.

DMSO compatible, flow directed or over the wire microcatheters are used for superselective catheterization of the arterial branches to the fistulous site. A gentle microinjection is performed to confirm the location of the site on a blank roadmap. If the pedicle is deemed suitable for embolization, a superselective angiogram is obtained. As previously described, similar characteristics are meticulously evaluated. The microcatheter is flushed with $10 \mathrm{ml}$ of normal saline. Onyx-18 is the typical embolysate used during the embolization. For high flow fistulas, Onyx-34 may be used. The Onyx solution must be shaken vigorously for $20 \mathrm{~min}$ to fully suspend the tantalum powder before it is used. Otherwise, sedimentation of the tantalum can cause inadequate opacification during infusion. DMSO and Onyx solutions are drawn into two separate DMSO resistant $1 \mathrm{ml}$ syringes. The deadspace of the microcatheter is slowly purged with DMSO, and the microcatheter hub is bathed with DMSO to form a meniscus. A slow steady injection should be about $0.1 \mathrm{ml} / \mathrm{min}$ and should not exceed $0.25 \mathrm{ml} / \mathrm{min}$ to avoid potential angiotoxicity of the solvent. Onyx is then injected for about $2 \mathrm{~min}$ to fill the microcatheter and to replace the DMSO in the deadspace under fluoroscopic roadmapping. This step is a critical part of the procedure to visualize and to prevent unintended infusion of the Onyx into normal vasculatures.

The blank roadmap technique is used during Onyx embolization. When reflux occurs proximally, the infusion is stopped for as long as 2 min to allow the Onyx to precipitate around the catheter. This strategy forms a plug at the catheter tip, thereby increasing the probability of antegrade flow. Whenever unwanted flow into non-targeted areas is observed, injections are stopped up to $2 \mathrm{~min}$. Each time, a subtracted fluoroscopic roadmap is refreshed to prevent confusion about the progression of the Onyx. This process may need to be repeated multiple times. This 'plug and push' technique can cast additional new arterial feeders through retrograde or antegrade artery to artery connections. Several milliliters of Onyx can be injected from a single pedicle. During these pauses, angiography is performed through the guide catheter to evaluate the residual flow to the DAVF and to avoid non-targeted embolization.

Onyx infusing into the sinuses can be tolerated if normal venous drainage does not depend on that venous segment. However, the procedure is stopped when an adequate Onyx cast is achieved or if retrograde reflux threatens to occlude en passage vessels or to trap the microcatheter. Retrieving the microcatheter after Onyx injection requires patience, gentle aspiration and constant tension on the catheter. Deflection of the Onyx cast is common, and gentle traction should be maintained as tension is slowly increased over several minutes. If the microcatheter cannot be removed, it is cut at the groin sheath.

Routine postoperative care is initiated. All patients are maintained normotensive in the intensive care unit. Heparinization is discontinued at the end of the endovascular procedure. Non-invasive postoperative imaging is performed only in patients with a worsening headache or new neurological symptoms.

\section{Results}

Of the 50 patients with 63 DAVFs who underwent transarterial Onyx embolization with and without adjuvant endovascular therapies, complete angiographic cure of 50 DAVFs (79\%) was obtained in 41 patients at the end of the procedure or at followup (range 3-25 months, median 5 months) (table 5). In 11 patients with 13 DAVFs (21\%) that were unsuccessfully treated via endovascular means, five patients with five DAVFs (8.3\%) required microsurgical disconnection for cure. After undergoing multiple ineffective staged embolization, two patients with four residual DAVFs (6.7\%) were treated with gamma knife

Table 5 Outcomes of 50 patients with 63 cranial DAVFs*

\begin{tabular}{ll}
\hline Outcome & No (\%) \\
\hline Total No of patients & 50 \\
$\begin{array}{l}\text { Total No of cranial DAVFs } \\
\text { Follow-ups (months) }\end{array}$ & 63 \\
$\quad$ Median & 5 \\
$\quad$ Range & $3-25$ \\
Successful endovascular cures & \\
$\quad$ Patients & $41(82)$ \\
$\quad$ DAVFs & $50(79)$ \\
Failed endovascular treatment & \\
$\quad$ Patients & $11(22)$ \\
$\quad$ DAVFs & $13(21)$ \\
Required microsurgery & \\
$\quad \begin{array}{l}\text { Patients } \\
\text { DAVFs }\end{array}$ & $5(10)$ \\
Radiosurgery & $5(8.3)$ \\
$\quad$ Patients & \\
$\quad$ DAVFs & $2(5)$ \\
\hline *Median follow-up 5 months (range 3-25 months). \\
DAVF, dural arteriovenous fistula.
\end{tabular}


radiosurgery after developing periprocedural endovascular complications. Three patients with three residual fistulas were lost to follow-up. One patient refused further angiography but remained clinically stable based on telephone conversations. In the 33 patients with 37 DAVFs that were treated exclusively via transarterial Onyx embolization in 39 sessions, an angiographic cure was obtained in 32 DAVFs (87\%) in 29 patients (table 6). Using the MMA as the primary embolization conduit, a $77 \%$ angiographic occlusion was achieved in 27 of the 35 DAVFs.

\section{Complications}

In the 76 embolizations, six (7.9\%) periprocedural complications occurred in four patients (8\%). Only one patient had a permanent complication (2\%). Patient No 1 developed a symptomatic stroke in the right medial temporal lobe causing temporary word finding difficulty that had resolved at follow-up after speech therapy. Patient No 2 developed persistent cranial nerve palsies with diplopia and dysphagia but had improved on follow-up. Patient No 3 had a very complex fistula with asymptomatic complications in three of the seven embolizations. The first complication occurred during the second session when a coil herniated into the straight sinus prompting the deployment of a Wingspan stent (Boston Scientific, Valencia, California, USA) to prevent sinus thrombosis of the deep venous system. During her fifth procedure, Onyx migrated into her left vertebral artery causing partial occlusion. The hypoplastic left vertebral artery was then sacrificed with additional coils and nBCA. During her last procedure, the microcatheter broke and migrated into the right internal carotid artery. The residual catheter was reoriented into the distal right common carotid artery via the Merci Retrieval Device (Concentric Medical Inc, Mountain View, California, USA). The microcatheter was then secured to the wall of the right common carotid artery via a Zilver stent (Cook Medical, Bloomington, Indiana, USA). This patient was asymptomatic after all three complications. In patient No 4, the microcatheter broke in his left common carotid artery, requiring placement of a Zilver stent. No neurological sequelae were observed.

\section{DISCUSSION}

DAVFs are uncommon intracranial vascular lesions but they can cause significant morbidity and mortality when they manifest with ICH or non-hemorrhagic neurological deficits. The risk for $\mathrm{ICH}$ is increased when lesions are in certain locations, such as the anterior fossa, and particularly when there is angiographic evidence of CVR. Endovascular techniques have evolved to become the main therapeutic option with the goal of obliterating the fistulous site and disconnecting CVR if present. The transarterial approach is slowly replacing the transvenous

Table 6 Success rates with transarterial Onyx alone and using the middle meningeal artery as a conduit

\begin{tabular}{ll}
\hline Procedure & No (\%) \\
\hline Transarterial Onyx alone & \\
Patients & 33 \\
DAVFs & 37 \\
Angiographic occlusion & \\
$\quad$ Patients & $29(88)$ \\
DAVFs & $32(87)$ \\
MMA & \\
DAVFs & 35 \\
Angiographic occlusion & $27(77)$ \\
\hline DAVF, dural arteriovenous fistula; MMA, middle meningeal artery.
\end{tabular}

approach for treating many types of DAVFs. The advent of Onyx and the preliminary promising results of its use in the transarterial treatment of DAVFs have paved new and exciting potential avenues for achieving higher curative rates.

The distinct characteristics of Onyx compared with nBCA improve the likelihood of obtaining angiographic occlusion of a DAVF through a single pedicle injection. The cohesive property of Onyx permits discontinuous injections on the order of minutes and allows serial angiographic analysis of the angioarchitecture of the lesion. In particular, small collaterals not visualized on initial angiography may become apparent with progressive infusion of Onyx. The ability to stop the infusion and to analyze the progression of the Onyx can minimize complications from unwanted infusion of the embolysate into potentially dangerous anastomoses or collaterals. The ability of Onyx to diffuse enables deeper penetration and occlusion of the fistulous network, and it can migrate retrograde into arterial feeders from other vessels through a single pedicle. Venous filling can be stopped temporarily without the threat of unintended migration of the Onyx into draining veins. These advantages allow exceptional control of the flow of Onyx and enable a large volume to be delivered via a single injection. The caveats used in embolizing cerebral AVMs are less relevant when treating DAVFs. Injection of a large volume of embosylate during embolization of an AVM can cause normal perfusion pressure breakthrough and subsequently ICH. Embolization of DAVFs is not associated with this risk.

However, transarterial Onyx embolization does have several disadvantages. The patient is exposed to prolonged radiation compared with the use of nBCA. Alopecia and radiation burns can follow. The delayed health risks of the prolonged exposure to radiation are unclear and difficult to assess. During embolization of cranial AVMs, Velat et al noted an increase in fluoroscopic time and procedural time. ${ }^{33}$ However, the advantage of fewer catheterizations when using Onyx compared with nBCA may balance this risk. Without a prospective comparison of these agents, we cannot conclude definitively whether one technique is associated with shorter fluoroscopic times. The cost of an Onyx procedure is significantly greater than a procedure performed with nBCA. Not only is the cost of each bottle of Onyx higher than $\mathrm{nBCA}$, a larger total volume of Onyx may be injected compared with nBCA. Despite its cohesive property, Onyx can adhere to the microcatheter and cause breakage, as demonstrated by two of our cases. Patience and gentle traction are required to remove the microcatheters at the end of the procedure. Breakaway microcatheters have been used successfully in Europe but are not yet available in the USA. The beneficial role of transarterial Onyx embolization must be balanced with the potential risks associated with the procedure.

Several studies have reported high success rates associated with using the MMA as the main embolizing arterial pedicle. Nogueira et al obtained complete angiographic cures in 11 of 12 patients using this method. ${ }^{31}$ Cognard et al reported complete cure of 15 of 18 patients by delivering Onyx in a single pedicle, particularly through the MMA. ${ }^{32}$ The arterial feeders in DAVFs, which are often branches of the external carotid artery (ECA) or dural based, can be notoriously tortuous and difficult to catheterize to reach the fistulous point. The MMA is often much easier to catheterize, even if its caliber is small compared with other larger, dilated branches of the ECAs. Another advantage of using the branches of the MMA is that they are anchored to the dura and calvarium. This feature, combined with the non-adhesive nature of Onyx, facilitates retrieval of the microcatheter at the end of an Onyx infusion and minimizes the risks 
of arterial rupture. In a prolonged injection, a significant volume of Onyx can be infused antegrade to occlude branches of the MMA as well as to fill retrograde through artery to vein to artery anastomoses with other feeders from the branches of the ECA. This technical advantage requires fewer catheterizations of the ECA to obtain an angiographic cure. Thus the MMA provides an excellent route for the delivery of Onyx to occlude the fistulous point and retrograde artery to fistula to artery occlusion of arterial feeders.

In the current senior authors' series, angiographic cures of DAVFs were achieved in $87 \%$ of patients undergoing embolization only with Onyx (figure 4). Subgroup analysis showed that by using the MMA as a conduit for primary Onyx embolization, we achieved angiographic cures of 27 of the 37 DAVFs. Two fistulas had ethmoidal feeders and received no supply from the MMA. Therefore, we achieved a $77 \%$ rate of angiographic cure using the MMA as an embolizing pedicle. Retrospectively, our success rate significantly improved in later cases compared with earlier ones, suggestive of a learning curve that has been augmented by experience.

The more important questions are why some DAVFs fail endovascular intervention and whether certain angiographic features can be used to increase the success of endovascular therapies. During our evaluation of the DAVFs that were not amenable to endovascular cures, we made several observations.
Several fistulas were incompletely occluded but blood flow was significantly slower. On follow-up angiography these fistula had progressed to complete thrombosis. In other cases, however, this progression did not occur and the discrepancy is puzzling. Most of the failed treatments occurred early in the series, suggesting that our technical skill with using Onyx improved with experience. Several failed DAVFs were complex (eg, torcular DAVFs) with bilateral anterior circulation supply as well as supply from the posterior circulation. These fistulas may require several staged embolizations and both transarterial and transvenous approaches. In our series, most of the intended staged embolizations were performed on consecutive days. The longest interval between stages was 10 days. This short interval did not appear to contribute to additional arterial recruitment.

In several cases that appeared to achieve angiographic success, the patients had developed fistulous connections to other sinuses at follow-up. Possibly, much smaller arterial feeders were not opacified because of flow limitation from small caliber vessels or from outflow limitation during this initial 'curative' stage. Conceivably, these arterial feeders might enlarge over time via a combination of flow diversion from pathological sinus occlusion and the angiogenic factors from hypoxic microenvironments. Interestingly, the initial DAVF of one patient drained into the left transverse-sigmoid sinus. Despite angiographic cure at the first session, she required two additional
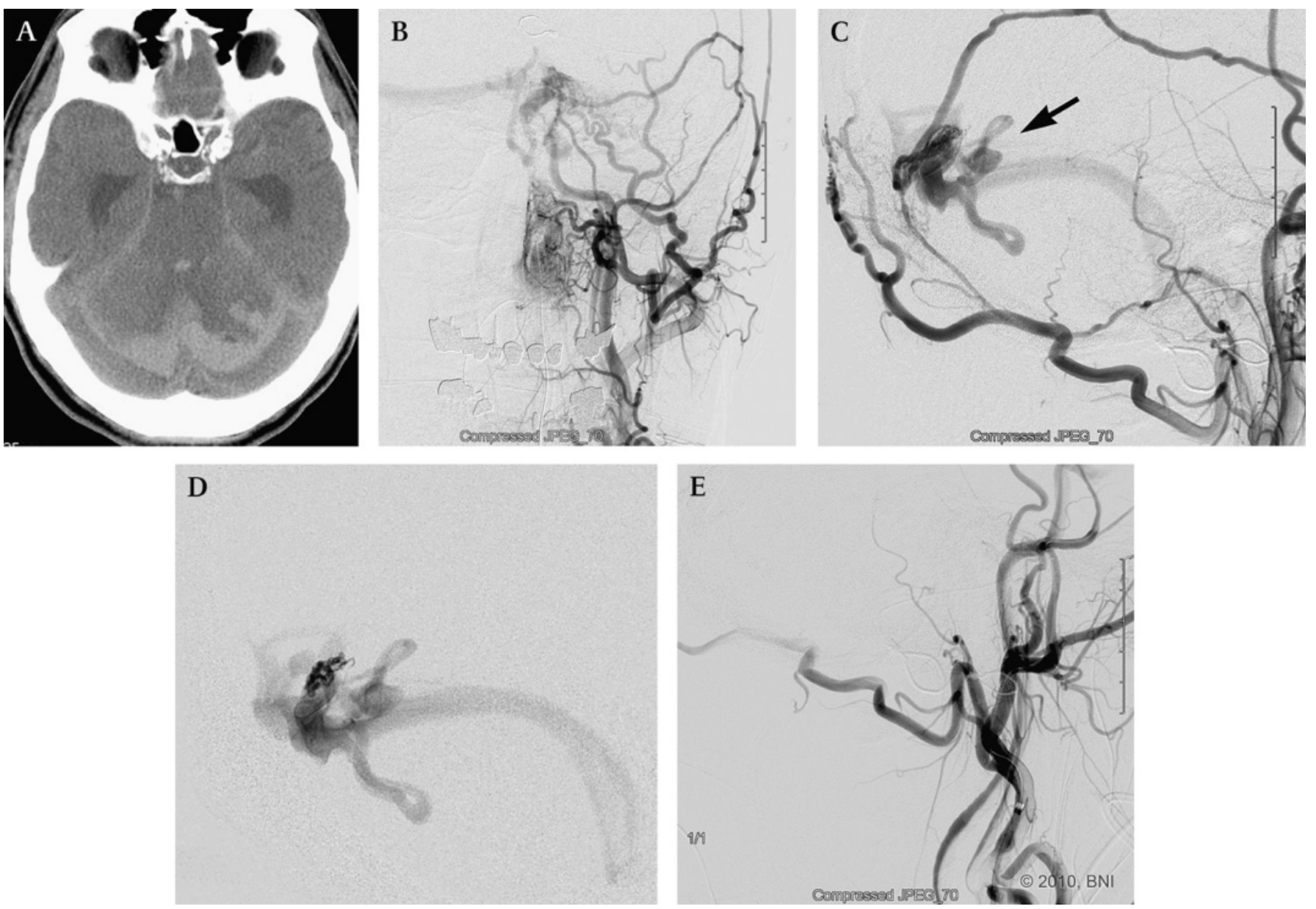

Figure 4 A 69-year-old patient with subarachnoid and intraventricular hemorrhage on CT of the head (A). Left external carotid artery (ECA) injection, anteroposterior (B) and lateral (C) views, showing an aggressive torcular dural arteriovenous fistula (DAVF) with arterial feeders from the middle meningeal artery (MMA), occipital artery and posterior auricular artery and venous drainage into the peritorcular cerebellar cortical veins (single arrow). Superselective distal left MMA injection (D) revealed similar angioarchitectural findings of the fistulous point. Final left ECA lateral angiography (E) showed complete occlusion of the fistula after a single infusion via the left MMA. (Used with permission from Barrow Neurological Institute.) 
embolizations for a recurrence, during which arterial flow through the same fistulous site was decreased significantly. A few months later, angiographic evaluation showed a new DAVF draining into the torcula. The previous left transverse-sigmoid sinus DAVF had progressed to occlusion. After improved but unsuccessful embolization of the torcular DAVF, new arterial feeders were recruited to drain into the right sigmoid sinus and later into the superior sagittal sinus a few months later. Why these new additional DAVFs formed is unclear but they may be related to venous outflow and angiogenic induced recruitment during the several months that had elapsed between treatments. In our other unsuccessful cases, four of the 13 DAVFs ultimately recruited or opacified feeders from tentorial branches of meningohypophyseal trunks. These vessels are often too small to catheterize and provide a low threshold for dangerous reflux proximally into the internal carotid artery.

Given that the goal of an embolization is permanent obstruction of the fistulous connection, the importance of durability of the embolic agent cannot be overlooked. Natarajan et al studied the durability of Onyx and found that the recanalization rate associated with their resected AVM specimens was $18 \%{ }^{34}$ This finding may implicate inadequate filling of Onyx in the vessel as the compound progressed via lamination in the vessels. However, it may be difficult to extrapolate their findings to the treatment of DAVFs because the durability of preoperative embolization of an AVM is less critical than in the management of DAVFs. Unfortunately, there are no definitive angiographic findings or calculations to determine the degree of casting in vessels embolized with Onyx. One can only surmise the extent of Onyx casting by the density visualized on subtracted angiographic views. Typically, contrast stasis is visible proximally when the embolized vessels are occluded, but whether recanalization or recruitment occurs later is unclear.

\section{CONCLUSION}

Transarterial embolization with Onyx represents a new paradigm for the treatment of DAVFs. Transarterial Onyx embolization of branches of the ECA, particularly the MMA, offers a high likelihood of a cure. The angioarchitecture of DAVFs, specifically those typically fed by dural based and extracranial arteries, allows a greater degree of reflux and thereby a greater degree of fistula penetration when infusions are prolonged. Transarterial Onyx provides a safe and effective method of embolization and is associated with few side effects and a very low rate of complications. However, long term follow-up is still needed to establish the efficacy of this agent.

\section{Competing interests None.}

Patient consent Obtained.

Ethics approval This manuscript complies with our institutional review board policy; however, patient identifiers have been anonymized.

Provenance and peer review Not commissioned; externally peer reviewed.

\section{REFERENCES}

1. Newton TH, Cronqvist S. Involvement of dural arteries in intracranial arteriovenous malformations. Radiology 1969:93:1071-8.

2. Newton TH, Greitz T. Arteriovenous communication between the occipital artery and transverse sinus. Radiology 1966:87:824-8.

3. Aminoff MJ, Kendall BE. Asymptomatic dural vascular anomalies. Br J Radiol 1973:46:662-7

4. Aminoff MJ. Vascular anomalies in the intracranial dura mater. Brain 1973:96:601-12.

5. Kosnik EJ, Hunt WE, Miller CA. Dural arteriovenous malformations. J Neurosurg 1974:40:322-9.
6. Obrador S, Soto M, Silvela J. Clinical syndromes of arteriovenous malformations of the transverse-sigmoid sinus. J Neurol Neurosurg Psychiatry 1975:38:436-51.

7. Djindjian R, Théron J, Merland J-J. Super-selective arteriography of the external carotid artery. In: Super-selective arteriography of the external carotid artery. New York, NY: Springer-Verlag, 1978.

8. Malik GM, Pearce JE, Ausman Jl, et al. Dural arteriovenous malformations and intracranial hemorrhage. Neurosurgery 1984;15:332-9.

9. Lasjaunias $\mathbf{P}$, Chiu M, ter Brugge $\mathrm{K}$, et al. Neurological manifestations of intracrania dural arteriovenous malformations. J Neurosurg 1986;64:724-30.

10. Awad IA, Little JR, Akarawi WP, et al. Intracranial dural arteriovenous malformations: factors predisposing to an aggressive neurological course. J Neurosurg 1990;72:839-50.

11. Piton J, Guilleux MH, Guibert-Tranier F, et al. Fistulae of the lateral sinus J Neuroradiol 1984:11:143-59.

12. Kerber CW, Newton TH. The macro and microvasculature of the dura mater Neuroradiology 1973;6:175-9.

13. Rothbart D, Awad IA, Lee J, et al. Expression of angiogenic factors and structural proteins in central nervous system vascular malformations. Neurosurgery 1996; 38:915-24.

14. Herman JM, Spetzler RF, Bederson JB, et al. Genesis of a dural arteriovenous malformation in a rat model. J Neurosurg 1995;83:539-45.

15. Terada T, Higashida RT, Halbach WV, et al. Development of acquired arteriovenous fistulas in rats due to venous hypertension. J Neurosurg 1994;80:884-9.

16. Borden JA, Wu JK, Shucart WA. A proposed classification for spinal and cranial dural arteriovenous fistulous malformations and implications for treatment. J Neurosurg 1995;82:166-79.

17. Cognard C, Gobin YP, Pierot L, et al. Cerebral dural arteriovenous fistulas: clinical and angiographic correlation with a revised classification of venous drainage. Radiology 1995;194:671-80

18. Satomi J, van Dijk JM, Terbrugge KG, et al. Benign cranial dural arteriovenous fistulas: outcome of conservative management based on the natural history of the lesion. J Neurosurg 2002;97:767-70.

19. Brown RD Jr, Wiebers DO, Nichols DA. Intracranial dural arteriovenous fistulae: angiographic predictors of intracranial hemorrhage and clinical outcome in nonsurgical patients. J Neurosurg 1994;81:531-8.

20. Davies MA, Terbrugge KG, Willinsky RA, et al. The natural history and management of intracranial dural arteriovenous fistulas, 2: aggressive lesions. Intervent Neuroradio 1997:64:303-11.

21. van Dijk JM, Terbrugge KG, Willinsky RA, et al. Clinical course of cranial dural arteriovenous fistulas with long-term persistent cortical venous reflux. Stroke 2002; 33:1233-6

22. Zipfel GJ, Shah MN, Refai D, et al. Cranial dural arteriovenous fistulas: modification of angiographic classification scales based on new natural history data. Neurosurg Focus 2009;26:E14.

23. Soderman M, Pavic L, Edner G, et al. Natural history of dural arteriovenous shunts Stroke 2008;39:1735-9.

24. Strom RG, Botros JA, Refai D, et al. Cranial dural arteriovenous fistulae: asymptomatic cortical venous drainage portends less aggressive clinical course. Neurosurgery 2009:64:241-7.

25. Duffau H, Lopes $M$, Janosevic $V$, et al. Early rebleeding from intracranial dural arteriovenous fistulas: report of 20 cases and review of the literature. J Neurosurg 1999:90:78-84.

26. Chandler HC Jr, Friedman WA. Successful radiosurgical treatment of a dural arteriovenous malformation: case report. Neurosurgery 1993:33:139-41.

27. Guo WY, Pan DH, Wu HM, et al. Radiosurgery as a treatment alternative for dural arteriovenous fistulas of the cavernous sinus. AJNR Am J Neuroradiol 1998;19:1081-7

28. Pollock BE, Nichols DA, Garrity JA, et al. Stereotactic radiosurgery and particulate embolization for cavernous sinus dural arteriovenous fistulae. Neurosurgery 1999:45:459-66.

29. Caragine LP, Halbach W, Dowd CF, et al. Parallel venous channel as the recipient pouch in transverse/sigmoid sinus dural fistulae. Neurosurgery 2003; 53:1261-6

30. Piske RL, Campos CM, Chaves JB, et al. Dural sinus compartment in dural arteriovenous shunts: a new angioarchitectural feature allowing superselective transvenous dural sinus occlusion treatment. AJNR Am J Neuroradiol 2005;26:1715-22.

31. Nogueira RG, Dabus G, Rabinov JD, et al. Preliminary experience with onyx embolization for the treatment of intracranial dural arteriovenous fistulas. AJNR Am J Neuroradiol 2008;29:91-7

32. Cognard C, Januel AC, Silva NA Jr, et al. Endovascular treatment of intracranial dural arteriovenous fistulas with cortical venous drainage: new management using Onyx. AJNR Am J Neuroradiol 2008;29:235-41.

33. Velat GJ, Reavey-Cantwell JF, Sistrom C, et al. Comparison of N-butyl cyanoacrylate and onyx for the embolization of intracranial arteriovenous malformations: analysis of fluoroscopy and procedure times. Neurosurgery 2008;63:0NS73-8.

34. Natarajan SK, Born D, Ghodke B, et al. Histopathological changes in brain arteriovenous malformations after embolization using Onyx or N-butyl cyanoacrylate. Laboratory investigation. J Neurosurg 2009:111:105-13. 